\title{
Cronin effect for protons and pions in high-energy pA collisions
}

\author{
A. H. Rezaeian and Zhun Lu \\ Departamento de Física y Centro de Estudios Subatómicos, Universidad Técnica \\ Federico Santa María, Casilla 110-V, Valparaíso, Chile
}

\begin{abstract}
Pions and protons production cross-sections are analyzed in proton-proton and proton-nucleus collisions at the RHIC energy at midrapidity. We employ the pQCD factorization scheme supplemented with the color-dipole formalism to investigate the Cronin effect. We calculate the broadening in the color-dipole approach for different centralities. Our main goal is to investigate, in a parameter-free manner within a unified framework, how much of the cronin effect for both pions and baryons comes from the transverse momentum broadening due to initial partons multi-scatterings. We conclude that final-state effects in pA collisions are important. Uncertainties in nuclear shadowing of various parton distributions and parton fragmentation functions are also discussed.
\end{abstract}

PACS:24.85.+p,25.75.-q, 13.60.Hb,13.85.Lg

Keywords: Quarks, gluons, and QCD in nuclear reactions, Relativistic heavy-ion collisions, Total and inclusive cross sections

\section{Introduction}

It is believed that $p+A$ collisions provide a decisive testing ground to distinguish between the initial- and final-state (plasma) effects in $A+A$ collisions and can be used as a baseline for jet-quenching models. The Cronin effect [1] (which is generally associated with the ratio of $p+A$ and $p+p$ cross sections, scaled by the number of collisions) in high-energy collisions has been the subject of renewed interest in recent years $[2,3,4,5,6,7,8,9]$. In order to pin down the role of parton energy loss effects in heavy ion collisions, a precise and firm understanding of the underlying dynamics of the Cronin and the shadowing effects in $p+A$ collisions is indispensable. 
There have been two very different approaches to explain the Cronin effect in $p+A$ collisions: the initial-state effects $[1,2]$ due to the broadening of the parton transverse momentum in the initial-state where the fragmentation of hard partons is assumed to occur outside the cold medium, and final-state effects [6] due to the recombination of soft and shower partons in the final-state. To understand the role of initial-state effects in the $p+A$ reactions, one should also understand the broadening of transverse momentum of a projectile parton propagating and interacting with a nuclear medium. A first principle calculation of the broadening of transverse momentum of partons is very complicated which involves apparently both soft and hard interactions. A common practice involves fitting to a given experiment and then extrapolation to another reaction [3]. However, such an approach is less reliable since broadening is not universal and depends on kinematics and reaction.

Here, we employ the pQCD factorization scheme supplemented with the colordipole formalism to investigate the Cronin effect for identified hadrons. The broadening is calculated in a parameter-free way in the color-dipole approach. Our modest goal is to investigate the role of initial-state effects in the observed Cronin ratio for both protons and pions in $p+A$ collisions and to learn how much of the effect comes from the broadening due to initial partons multiscatterings. One of the uncertainties in such a calculation from the outset is due to our lack of knowledge about the baryon fragmentation functions. We investigate the implication of the uncertainties among various nuclear parton distributions and parton fragmentation functions and the role of parton primordial transverse momentum on the Cronin effect.

One of the stunning experimental observations in both $p+A$ and $A+A$ collisions has been the much larger magnitude of the Cronin effect on baryons compared to mesons at the intermediate transverse momentum, the so-called baryon/meson anomaly in high energy nuclear collisions [10,11]. There have been different approaches to understand this phenomenon [6,12]. But a clear explanation is still lacking. Here, we compute the protons/pions ratio in $p+p$ and $p+A$ collisions. We also address if the underlying production mechanism of baryons and mesons are the same.

This paper is organized as follows: In Sec 2, we discuss hadron production in $p+p$ collisions. In Sec. 3, we introduce the main elements of our computation hadron spectra in $p+A$ collisions. In particular, we discuss how to compute broadening in the color-dipole formalism. Sec. 4 summarizes our work. 


\section{Particle production in $p+p$ collisions}

High- $p_{T}$ inclusive particle production in hadron scattering may be described by collinear pQCD factorization. However, in the derivation of the collinear factorization theorems, one applies to the hard scattering the approximation that the transverse momentum of the incoming parton can be neglected with respect to the transverse momentum generated in the scattering, and one also neglects the transverse momentum generated in the fragmentation. When the transverse momentum of the incoming partons is in the order of hard scale $Q$, the errors in the collinear approximations can be compensated by a correct treatment of higher order corrections to the hard scattering. However, for low transverse momentum compared with $Q$, in collinear factorization there is no precise compensation from higher order correction and one should not neglect the transverse momentum of the incoming partons.

Moreover, it is well known that the curvature of the hadron spectrum can be corrected by considering intrinsic transverse momentum $k_{T}$ for the colliding partons. It was observed that significant parton intrinsic transverse momentum is needed for describing data of Drell-Yan dilepton production [13], direct photon production [14,15] and heavy quark production [16]. The origin of such a large primordial transverse momentum still needs a clear explanation. Nevertheless, some higher order pQCD corrections and soft gluon radiation corrections are effectively embodied in the primordial transverse momentum distribution. There are also some spin-dependent effects measured by experiments, such as single spin asymmetry, which cannot be explained by collinear factorization in all orders of QCD. The only way to produce these non-zero

effects is with the presence of parton intrinsic transverse momentum [17] or by considering the higher-twist contributions.

There exists many ways of incorporating the parton intrinsic transverse momentum phenomenologically, and we choose for simplicity a $k_{T}$ smearing of the cross section to approximate this effect via unintegrated parton distributions. This pQCD-improved parton model based on factorization should not be mixed with the collinear factorization. Notice that we do not aim at comparing different types of factorizations to claim which one is better or not. Each of them can be applied to some specific processes and specific kinematical region. Here, we prefer to use a kind of $k_{T}$-factorization which is flexible enough in the case of $p+A$ collisions.

By assuming the validation of the factorization in high- $p_{T}$ particle production, and considering the intrinsic transverse momenta of the initial partons, the differential cross section in $p+p$ collision can be written as [18], 


$$
\begin{aligned}
\frac{d \sigma^{p p \rightarrow h+X}}{d y d^{2} p_{T}} & =\sum_{i j n l} \int d x_{i} d x_{j} d^{2} k_{i T} d^{2} k_{j T} f_{i / p}\left(x_{i}, Q^{2}\right) \mathcal{G}_{p}\left(k_{i T}, Q^{2}\right) \\
& \times f_{j / p}\left(x_{j}, Q^{2}\right) \mathcal{G}_{p}\left(k_{j T}, Q^{2}\right) K \frac{d \sigma}{d \hat{t}}(i j \rightarrow n l) \frac{D_{h / n}\left(z_{n}, Q^{2}\right)}{\pi z_{n}}
\end{aligned}
$$

where the $K$ factor which is in general $\sqrt{s}$ and scale dependent accounts for the contribution of the NLO corrections (for the Cronin ratio which is the main subject of this paper, the $K$ factor drops out and is not more important). $f_{i / p}\left(x_{i}, Q^{2}\right)$ is the parton distribution functions (PDF) of the colliding protons, which depend on the light-cone momentum fractions $x_{i}$ and the hard scale $Q$. The function $D_{h / n}\left(z_{n}, Q^{2}\right)$ is the fragmentation function (FF) of parton $n$ to the final hadron $h$ with a momentum fraction $z_{n}$. The differential cross section $\frac{d \sigma}{d \hat{t}}(i j \rightarrow n l)$ of the hard process $a\left(k_{i}\right)+b\left(k_{j}\right) \rightarrow c\left(k_{n}\right)+d\left(k_{l}\right)$ can be calculated perturbatively(in the running coupling $\alpha_{s}\left(Q^{2}\right)$ with scale parameter $\Lambda$ equal to pion mass), in terms of the following Mandelstam variables

$$
\begin{aligned}
& \hat{s}=\left(k_{i}+k_{j}\right)^{2}=x_{i} x_{j} s+\frac{k_{i T}^{2} k_{j T}^{2}}{x_{i} x_{j} s}-2 \boldsymbol{k}_{i T} \cdot \boldsymbol{k}_{j T}, \\
& \hat{t}=\left(k_{i}-k_{n}\right)^{2}=-\frac{1}{z_{n}}\left(x_{i} \sqrt{s} p_{T} e^{-y}+\frac{k_{i T}^{2}}{x_{i} \sqrt{s}} p_{T} e^{y}-2 \boldsymbol{k}_{i T} \cdot \boldsymbol{p}_{T}\right), \\
& \hat{u}=\left(k_{j}-k_{n}\right)^{2}=-\frac{1}{z_{n}}\left(x_{j} \sqrt{s} p_{T} e^{y}+\frac{k_{j T}^{2}}{x_{j} \sqrt{s}} p_{T} e^{-y}-2 \boldsymbol{k}_{j T} \cdot \boldsymbol{p}_{T}\right),
\end{aligned}
$$

and $\sqrt{s}$ is the center of mass energy of the incoming $p+p$ system, $y$ is the rapidity of the produced hadron defined as $y=\frac{1}{2} \ln \left(E+p_{L}\right) /\left(E-p_{L}\right)$. To avoid the divergence of the partonic cross-sections when one of the Mandelstam variables approaches to zero, we apply following replacement

$$
\hat{s} \rightarrow \hat{s}+2 \mu^{2}, \quad \hat{t} \rightarrow \hat{t}-\mu^{2}, \quad \hat{u} \rightarrow \hat{u}-\mu^{2},
$$

with $\mu=0.8 \mathrm{GeV}$. This replacement will not change the partonic cross-sections at large $\hat{s}, \hat{t}$ and $\hat{u}$. Another advantage of this replacement is that the relation $\hat{s}+\hat{t}+\hat{u}=0$ is always satisfied.

The function $\mathcal{G}_{p}\left(k_{a T}\right)$ describes the distribution of intrinsic transverse momenta carried by partons. In phenomenological applications, a Gaussian form for $\mathcal{G}_{p}\left(k_{T}\right)$ is usually used:

$$
\mathcal{G}_{p}\left(k_{T}\right)=\frac{\exp \left(-k_{T}^{2} /\left\langle k_{T}^{2}\right\rangle\right)}{\pi\left\langle k_{T}^{2}\right\rangle},
$$

where $\left\langle k_{T}^{2}\right\rangle$ is the square of the 2-dimensional RMS width of the $k_{T}$ distribution for one parton, which is related to the square of the 2-dimensional average of the absolute value of $k_{T}$ of one parton through $\left\langle k_{T}^{2}\right\rangle=4\left\langle k_{T}\right\rangle^{2} / \pi$. For simplicity, we assume $\left\langle k_{T}^{2}\right\rangle$ to be independent of $Q^{2}$. 




Fig. 1. Cross section of $p+p \rightarrow \pi^{0}+X$ at RHIC energy $\sqrt{s}=200 \mathrm{GeV}$ for AKK08 and Kretzer fragmentation functions. The error bars are the total uncertainties. Data are from the PHENIX collaboration [23].

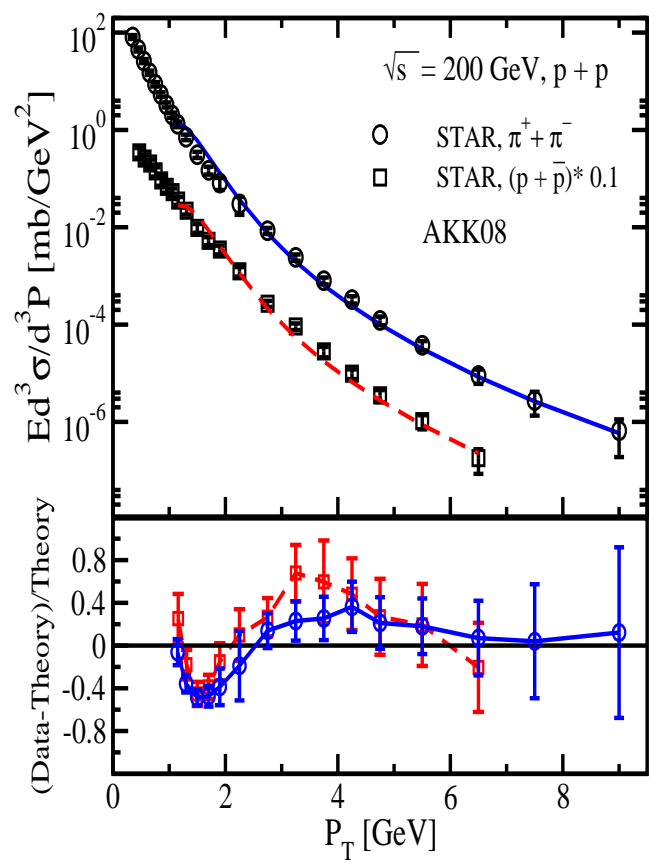

Fig. 2. Cross section of $p+p \rightarrow \pi^{+}\left(\pi^{-}\right)+X$ and $p+p \rightarrow p(\bar{p})+X$ at RHIC energy $\sqrt{s}=200 \mathrm{GeV}$. For theory curves we use the parameter set with AKK08 fragmentation function, see the text. The error bars are the total statistical and systematic uncertainties. Data are from the STAR collaboration [10]. 


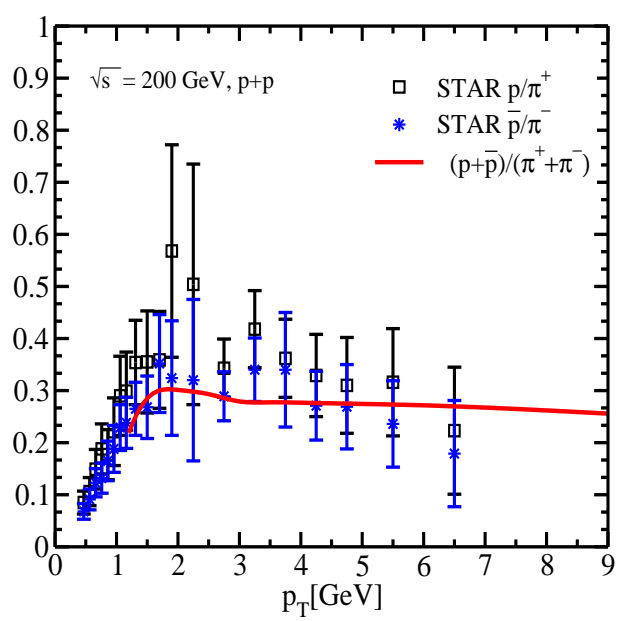

Fig. 3. Ratio of $(p+\bar{p}) /\left(\pi^{+}+\pi^{-}\right)$at RHIC energy $\sqrt{s}=200 \mathrm{GeV}$ for midrapidity with AKK08 FF. Data are for ratio of $\bar{p} / \pi^{-}$and $p / \pi^{+}$at midrapidity $(|y|<0.5)$. The error bars are the total statistical and systematic uncertainties. Data are taken from Ref. [10].

With the factorization form shown in Eq. (1), we calculate the cross section of $p+p \rightarrow \pi^{0}+X$ at RHIC for $\sqrt{s}=200 \mathrm{GeV}$. For the parton distribution $f_{i / p}\left(x, Q^{2}\right)$ used in our calculations we adopt the newest MRST PDF [19]. For the FF $D_{h / n}\left(z_{n}, Q^{2}\right)$, we first apply the parametrization by Kretzer [20]. However, Kretzer FFs do not give parton FFs to protons. Therefore, we also consider AKK08 parametrization [21] of FFs which give parton FFs to pions and $(p+\bar{p})$. In both cases of calculations with Kretzer and AKK08 FFs, we will take $\left\langle k_{T}^{2}\right\rangle=2 \mathrm{GeV}^{2}$ and $Q=p_{T}$ for the scale $Q$ of the hard process. Notice also that different value of $\left\langle k_{T}^{2}\right\rangle$ has been used in different approaches $[9,22]$. We take the $K$-factor $K=2.5,1.5$ for the cases of Kretzer and AKK08 parametrization of FFs, respectively, which gives a good approximation of the higher order contribution in the $p_{T}$ region of interest. With the settings mentioned above, we are able to reproduce the pions $\left(\pi^{0}, \pi^{+}+\pi^{-}\right)$productions data from the STAR and PHENIX collaborations $[23,10]$ for $p+p$ collisions. The discrepancy between theory and the data is within $40 \%$, see Figs. $(1,2)$. The setting with AKK08 FF also gives good predictions for protons productions in $p+p$ collisions in accordance with data from the STAR collaboration [10], see Fig. (2).

In Fig. (3), we show experimental data from the STAR collaboration for ratio of $\bar{p} / \pi^{-}$and $p / \pi^{+}$at midrapidity. The ratio of $(p+\bar{p}) /\left(\pi^{+}+\pi^{-}\right)$at midrapidity obtained with AKK08 FF, seems to be consistent with the experimental data for $\bar{p} / \pi^{-}$and $p / \pi^{+}$. 


\section{$3 p+A$ collisions and Cronin effect}

Multiple interactions of projectile partons in the target may proceed coherently or incoherently. In the former case the multiple interaction amplitude is a convolution of single scattering amplitudes, and in the latter case one should convolute differential cross sections, rather than amplitudes. The underlying mechanisms of the multiple particle interactions and particle productions are controlled by the coherence length [24],

$$
l_{c} \simeq \frac{\langle z\rangle \sqrt{s}}{m_{N} p_{T}},
$$

where $p_{T}$ is the transverse momentum of the fragmented hadron at midrapidity, and $m_{N}$ is the nucleon mass. For pion production, the average momentum fraction $\langle z\rangle$ in the FFs is about $0.4-0.6$ in the range of $2 \leq p_{T}(\mathrm{GeV}) \leq 8$. For a coherence length which is shorter than the typical internucleon separation $l_{c} \lesssim R_{A}$ (where $R_{A}$ denotes the nuclear radius), the projectile interacts incoherently. At the RHIC energy, $\sqrt{s}=200 \mathrm{GeV}$, within intermediate $p_{T}$ we are almost in the transition regimes between the short- and long-coherence limit for more central collisions, and at higher $p_{T}$ we are in the short-coherence length (SCL) limit.

Here, we resort to a simple scheme assuming that the pQCD factorization is valid at the RHIC energy at the midrapidity. We assume that the high- $p_{T}$ hadrons are mainly originated from projectile's partons whose transverse momentum is broadened by partons multi-scattering via gluons exchange. The broadening is computed in a parameter-free manner in the color dipole approach. The single inclusive particle cross section in minimum-biased $p(d)+A$ collisions can be written as

$$
\begin{aligned}
\frac{d \sigma^{p(d) A \rightarrow h+X}}{d y d^{2} \vec{p}_{T} d^{2} \vec{b}} & =\sum_{i j n l} \int d x_{i} d x_{j} d^{2} k_{i T} d^{2} k_{j T} T_{A}(b) f_{i / p(d)}\left(x_{i}, Q^{2}\right) \tilde{\mathcal{G}}_{p}\left(k_{i T}, b, Q^{2}\right) \\
& \times \tilde{f}_{j / A}\left(x_{j}, b, Q^{2}\right) \mathcal{G}_{p}\left(k_{j T}, Q^{2}\right) K \frac{d \sigma}{d \hat{t}}(i j \rightarrow n l) \frac{D_{h / n}\left(z_{n}, Q^{2}\right)}{\pi z_{n}}
\end{aligned}
$$

where $T_{A}(b)$ is the nuclear thickness function normalized to $\int d^{2} b T_{A}(b)=A$. We will use the Woods-Saxon nuclear profile for $T_{A}(b) . \tilde{f}_{j / A}\left(x_{j}, b, Q^{2}\right)$ denotes the parton distribution function (Npdf) in the target nuclei (with atomic number $A$ and charge number $Z$ ) which can be parametrized in a factorizable form

$$
\tilde{f}_{j / A}\left(x_{j}, b, Q^{2}\right)=R_{j / A}\left(x, b, Q^{2}\right)\left[\frac{Z}{A} f_{j / p}\left(x, Q^{2}\right)+\left(1-\frac{Z}{A}\right) f_{j / n}\left(x, Q^{2}\right)\right],
$$

in terms of parton distribution in a nucleon $f_{j / n}\left(x, Q^{2}\right)$ and the nuclear mod- 
ification function factor $R_{j / A}\left(x, b, Q^{2}\right)$. We will show the results of our calculation using three different Npdfs: EKS [25] and HKN [26] which are impactparameter independent but the $Q^{2}$-scale dependent via DGLAP evolution equation and HIJING-new [27] which is impact-parameter dependent.

Initial/final state broadening of the projectile/ejectile partons is effectively taken into account via a modification of the primordial transverse momentum distribution,

$$
\tilde{\mathcal{G}}_{p}\left(k_{i T}, b, Q^{2}\right)=\frac{d \sigma^{i=q}(q A \rightarrow q X)}{d^{2} \vec{k}_{i T}}(x, b),
$$

where the transverse momentum distribution of partons after propagation through nuclear matter of thickness $T_{A}(b)$ is subjected to the broadening and computed in terms of the propagation of a $q \bar{q}$ color dipole through the target nucleus [28]

$$
\begin{aligned}
\frac{d \sigma^{i=q}(q A \rightarrow q X)}{d^{2} \vec{p}_{T}}(x, b) & =\frac{1}{(2 \pi)^{2}} \int d^{2} \vec{r}_{1} d^{2} \vec{r}_{2} e^{i \vec{p}_{T} \cdot\left(\vec{r}_{1}-\vec{r}_{2}\right)} \Omega_{i n}^{q}\left(\vec{r}_{1}, \vec{r}_{2}\right) \\
& \times e^{-\frac{1}{2} \sigma_{q \bar{q}}\left(\vec{r}_{1}-\vec{r}_{2}, x\right) T_{A}(b)}
\end{aligned}
$$

where $\Omega_{i n}^{q}\left(\vec{r}_{1}, \vec{r}_{2}\right)$ is the density matrix which describes the impact parameter distribution of the quark in the incident hadron,

$$
\Omega_{i n}^{q}\left(\vec{r}_{1}, \vec{r}_{2}\right)=\frac{\left\langle k_{T}^{2}\right\rangle}{\pi} e^{-\frac{1}{2}\left(r_{1}^{2}+r_{2}^{2}\right)\left\langle k_{T}^{2}\right\rangle}
$$

where $\left\langle k_{T}^{2}\right\rangle$ denotes the mean value of the parton primordial transverse momentum squared. For a projectile gluon $(i=g)$ we replace the $q \bar{q}$ dipole with a $g g$ one which can be written again in terms of $q \bar{q}$ dipole via a Casimir factor $\sigma_{g g}=\frac{9}{4} \sigma_{q \bar{q}}$. The appearance of the dipole cross section in Eq. (11), is the result of a product of the amplitude and the time-conjugated one, which describe the quarks with different impact parameters. Clearly, the object participating in the scattering is not a $q \bar{q}$ dipole but rather a single colored parton. To simplify the calculations we assumed that the initial and final partons are the same, so the total nuclear thickness $T_{A}(b)$ contributes to broadening.

We take for the dipole cross-section in Eq. ( 11) for the case of a projectile quark $(i=q)$, the popular saturation model of Golec-Biernat and Wüsthoff [29]: $\sigma_{q \bar{q}}(x, \vec{r})=\sigma_{0}\left(1-\exp \left(-r^{2} / R_{0}^{2}\right)\right)$ where the parameters, fitted to DIS HERA data at small $x$, are given by $\sigma_{0}=23.03 \mathrm{mb}, R_{0}=0.4 \mathrm{fm} \times\left(x / x_{0}\right)^{0.144}$, where $x_{0}=3.04 \times 10^{-4}$. This parametrization gives a good description of DIS data at $x<0.01[29]$.

The broadening in this scheme depends on the impact parameter $b$, the transverse momentum of incident parton $k_{T}$, and also on the target Bjorken $x$. Note 


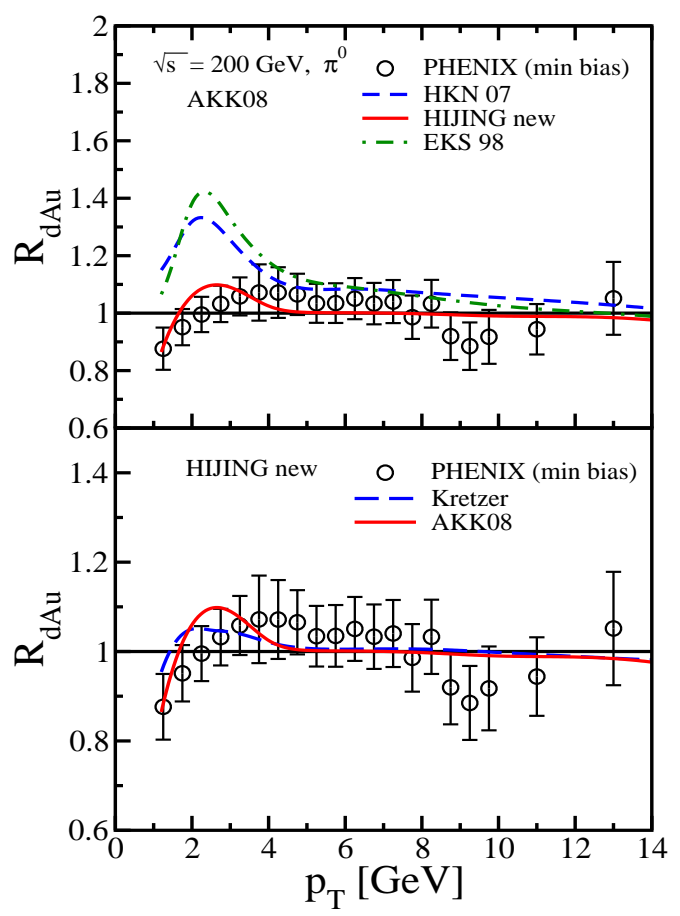

Fig. 4. The mini-biased Cronin ratio $R$ for $\pi^{0}$ production at RHIC energy $\sqrt{s}=200$ $\mathrm{GeV}$. All curves are at midrapidity. In the upper panel, we show $R_{d A u}$ with AKK08 FF for different nuclear PDF. In the lower panel, we show $R_{d A u}$ with HIJING-new nuclear PDF for different FFs. The normalization uncertainty on the $p+p$ reference of $9.7 \%$ is not included here. The error bars are the total statistical and systematic uncertainties. Data are taken from Ref. [23].

that in Eq. (8), the summation over index $i, j, n, l$ for quarks and gluons are different, not only because the corresponding pdfs and FFs are different, but also because the broadening of the projectile quarks and gluons are different.

The $K$-factor in Eq. (8) and the parton primordial transverse momentum squared $\left\langle k_{T}^{2}\right\rangle$ in Eqs. $(8,12)$ are taken the same value fixed in $p+p$ collisions. Therefore, all the phenomenological parameters in the master equation (8) are already fixed in reactions different from $p(d)+A$ collisions.

The nuclear modification factor $R_{p A}$ defined as ratio of $p+A$ to $p+p$ cross sections normalized to the average number of binary nucleon collisions,

$$
R_{p A}=\frac{\frac{d \sigma^{p A \rightarrow h+X}}{d y d^{2} p_{T}}}{\left\langle N_{\text {binary }}\right\rangle \frac{d \sigma^{p p \rightarrow h+X}}{d y d^{2} p_{T}}},
$$

where $\left\langle N_{\text {binary }}\right\rangle$ is the average number of geometrical binary collisions which is calculated according to the Glauber model [30] for different centrality.

In Fig. (4) (upper panel), we show mini-biased $R_{d A u}$ for $\pi^{0}$ production at the RHIC energy for deuteron-Gold collisions with AKK08 FF for various Npdfs. 


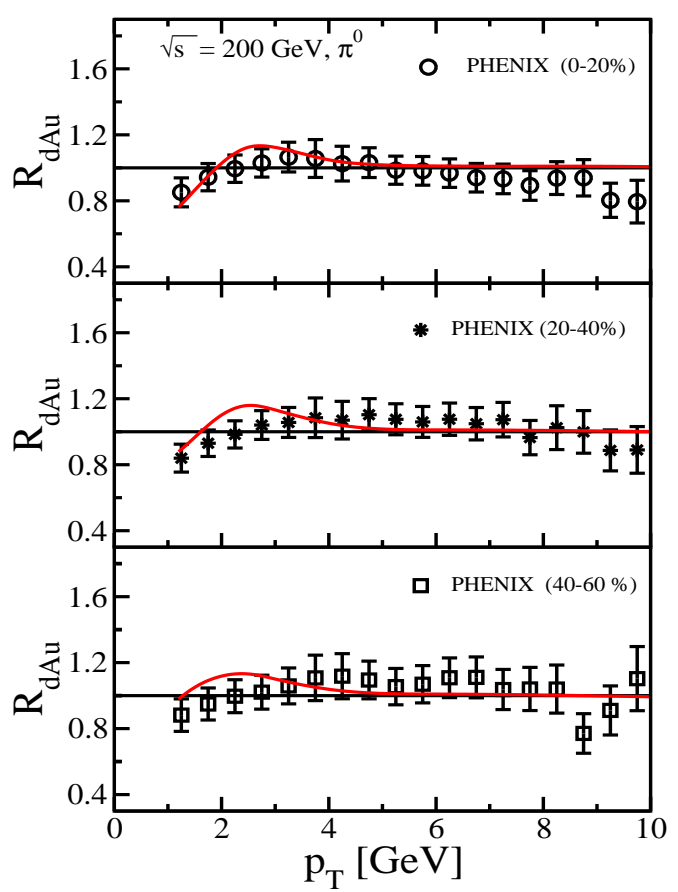

Fig. 5. The Cronin ratio $R$ for $\pi^{0}$ production at RHIC energy $\sqrt{s}=200 \mathrm{GeV}$ at different centralities. All curves are at midrapidity with AKK08 FF and HIJING-new nuclear PDF. Data are taken from Ref. [23].

It is obvious that the uncertainty among various Npdfs at small $p_{T}$ in the shadowing region leads to rather sizable different Cronin ratios. The position of the $R_{d A u}$ peak in $p_{T}$ does not seem to vary that much among various Npdfs.

In Fig. (4) (lower panel), we show $R_{d A u}$ for $\pi^{0}$ production with HIJING-new Npdf for different FFs. As one may expect both considered fragmentation functions (Kretzer and AKK08 FFs) give very similar results for $R_{d A u}$, since we assumed no medium modification for FFs. Notice also that the energy loss is less important at the RHIC energy for $d+A u$ collisions, although it might be important at lower energies at SPS $[5,31]$.

One should note that the nuclear interactions that lead to shadowing are also the source of parton momentum broadening. We incorporate the shadowing effect and the other nuclear medium modification of the partons through the Npdfs Eq. (9). In principle, at high-energy the $q \bar{q}$ dipole cross-section used for obtaining the broadening is also subjected to the multi-Pomeron fusion effect in the presence of nuclear medium. However, we have already incorporated the shadowing effect in the Npdf and including the shadowing effect into the dipole cross-section may lead to double counting. One should also note that the onset of gluon shadowing and its magnitude are still debatable, see Ref. [32] and references therein.

In Fig. (5), we show the Cronin ratio at different centralities at midrapidity. 


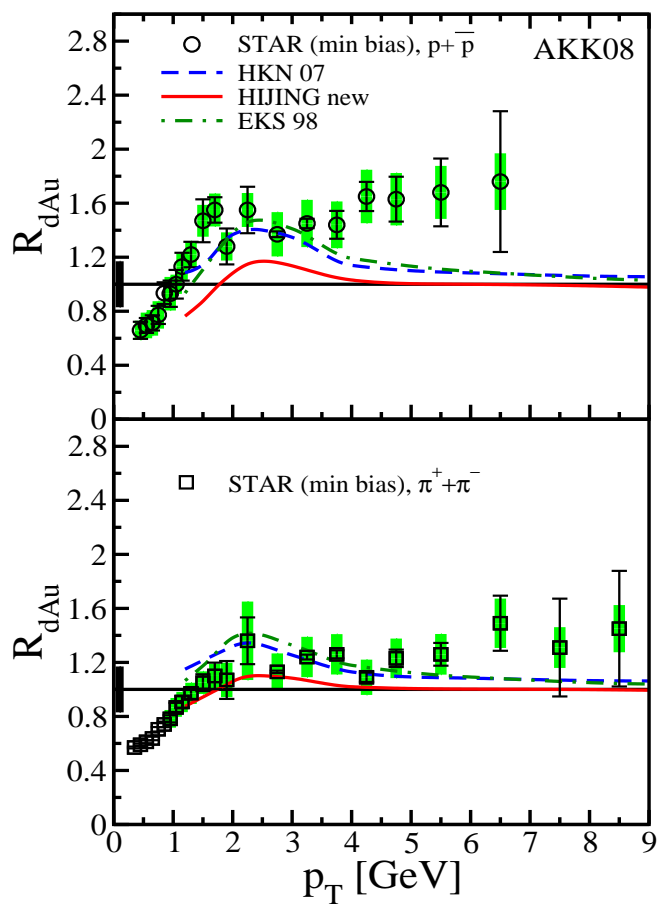

Fig. 6. The mini-biased Cronin ratio $R$ for charged pions $\pi^{+}+\pi^{-}$and baryons $p+\bar{p}$ productions at RHIC energy $\sqrt{s}=200 \mathrm{GeV}$ at midrapidity with AKK08 FF for various Npdfs. The experimental data are for $|y|<0.5$. The shaded bands around 1 (of the order of $17 \%$ ) corresponds to the error due to uncertainties in estimating the number of binary collisions in minimum bias. Data are taken from Ref. [10].

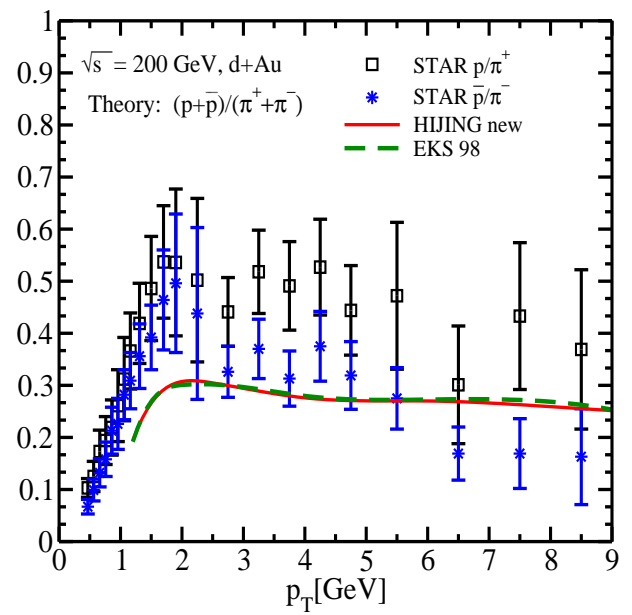

Fig. 7. The mini-biased ratio of $(p+\bar{p}) /\left(\pi^{+}+\pi^{-}\right)$for $d+A u$ collisions at RHIC energy $\sqrt{s}=200 \mathrm{GeV}$ for midrapidity with AKK08 FF and various Npdfs. Data are for ratio of $\bar{p} / \pi^{-}$and $p / \pi^{+}$at midrapidity $(|y|<0.5)$ for minimum bias $d+A u$ collisions. The error bars are the total statistical and systematic uncertainties. Data are taken from Ref. [10].

We used for all curves AKK08 FF and HIJING-new Npdf. It is obvious that generally the centrality dependence is rather weak in accordance with the PHENIX data [23]. 


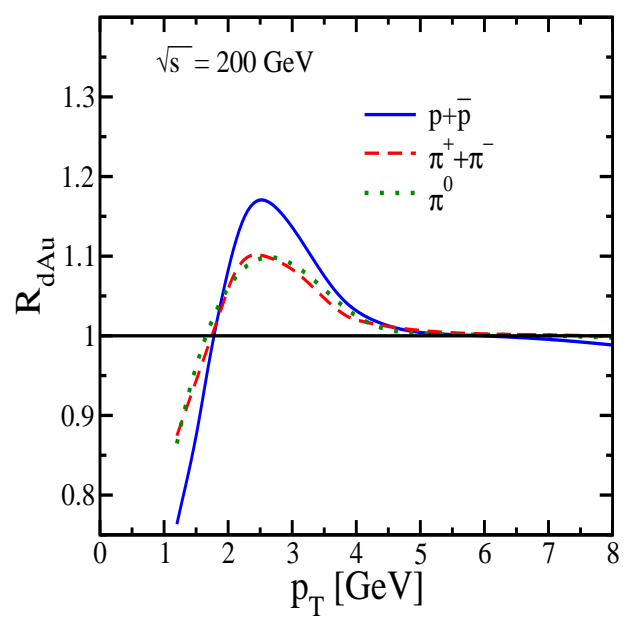

Fig. 8. The mini-biased Cronin ratio for pions and protons at midrapidity for the RHIC energy with AKK08 FF and HIJING-new Npdf.

In Fig. (6), we show the mini-biased $R_{d A u}$ at midrapidity for charged pions $\pi^{+}+\pi^{-}$and baryons $p+\bar{p}$ at midrapidity with AKK08 FF for various Npdfs. It is seen from Figs. $(4,5,6)$ that our results obtained with HIJING-new Npdf agree with the Cronin data for both $\pi^{0}$ and charged pions $\pi^{+}+\pi^{-}$. However, the same setting does not seem to be in a good agreement with the Cronin data for baryons in $p+A$ collisions although it gives a good description of baryons cross-section in $p+p$ collisions. Taking the experimental data at face value, the discrepancy between the theory and experimental data seems to persist up to $p_{T}=6.5 \mathrm{GeV}$. A number of studies have recently found that recombination is more important than fragmentation at small and moderate $p_{T}$ at midrapidity in heavy-ion collisions at the RHIC energy $\sqrt{s}=200 \mathrm{GeV}$ [6,33]. Therefore, a precise experimental measurement of the Cronin ratio for baryons at higher $p_{T}>7 \mathrm{GeV}$ is essential in order to reveal the underlying mechanism of hadron production in the cold nuclear matter. This deviation is also seen from Fig. (7) where similar to Fig. (3), we plot $(p+\bar{p}) /\left(\pi^{+}+\pi^{-}\right)$ ratio in $d+A u$ collisions at midrapidity for various Npdfs. From Fig. (7), it is obvious that baryons/pions ratio in $p+A$ collisions is not sensitive to the shadowing effects in contrast to $R_{p A}$. To conclude, these discrepancies indicate that not entire Cronin effect in $p+A$ collisions comes from the broadening due to initial partons multi-scatterings, and final-state effects which are not included here, are also important.

In Fig. (8), we compare the mini-biased Cronin ratio for pions and protons at midrapidity for the RHIC energy with AKK08 FF and HIJING-new Npdf. It is obvious that the Cronin ratios for baryons and mesons coming from initialstate effects are not significantly different. 


\section{Summary and final remarks}

In this paper, we studied pions and baryons productions in $p+p$ and $d+$ $A u$ collisions at the RHIC energy. Having a successful description of pions and protons productions in $p+p$ collisions, we showed that the observed Cronin ratio for pions in $d+A u$ collisions can be fairly described by transverse momentum broadening due to initial partons multi-scatterings. But the same mechanism seems to underestimate the observed Cronin ratio for protons in $d+A u$ collisions. We stress that all phenomenological parameters in our model are fixed in reactions different from $d+A u$ collisions. This discrepancy might indicate that initial state-effects (assuming that the fragmentation of hard partons occurs outside of medium) might not be totally accountable for the observed Cronin effect for baryons in $p+A$ collisions and the separation of partons into two non-interacting components, soft and hard, might be an oversimplification. It is also possible that the baryons productions mechanism in cold nuclear medium to be different from pions productions.

A similar approach to this paper, was taken by Kopeliovich et al. in Ref. [5] to calculate the mini-biased Cronin ratio for $\pi^{0}$. In this paper, we extended their study by calculating the Cronin effect for the charged pions and protons at different centralities. One of the major difference between our study with Ref. [5] is the way that the shadowing is included. As we already mentioned at moderate $p_{T}$ at the energy of RHIC, we are in the transition regime between the short- and long-coherence limits. Here, we assumed that the pQCD factorization is still valid in the transition region, and we included the shadowing effect in the conventional way via the nuclear parton distribution. We also studied the effect of various available nuclear parton distributions. However, in Ref. [5] no shadowing was included in Npdf. While the Cronin ratio was obtained by a linear interpolation between two Cronin ratios obtained in two different schemes of the short- and long-coherence limits. We also tried their prescription in order to investigate the pions and protons productions in $d+A$ collisions. We found similar results to those obtained in our approach which leads us again to the same conclusion that baryons and pions productions mechanism in high-energy $p+A$ collisions may be different and final-state effects are important [6,33]. Nevertheless, given rather large experimental uncertainties further studies are needed in order to make a final verdict.

Notice that our prescription is not reliable at forward rapidities. The reason is due to the importance of the valence quarks contribution which are not incorporated in the color dipole picture. It might be tempting to assume that forward rapidity region should be valid domain of our scheme since the Bjorken $x_{2}$ of target is small, and the color-dipole approach which is based on Pomeron-exchanges should be at work. However, one should note that at this region $x_{1} \rightarrow 1$ and as a consequence the energy conservation put a restricted 
constraint on the particle productions and therefore valence quarks become very important [8].

\section{Acknowledgments}

AHR would like to thank Boris Kopeliovich, Eugene Levin and Ivan Schmidt for valuable discussions. AHR wishes also to thank Hans-Jürgen Pirner for asking a question which led to this investigation. The authors are very grateful from Bedanga Mohanty and Simon Albino for useful communication. This work was supported in part by Conicyt (Chile) Programa Bicentenario PSD91-2006, Fondecyt (Chile) grants 1070517 and Project of PBCT (Chile) No. ACT-028.

\section{References}

[1] J. W. Cronin et al., Phys. Rev. D11, 3105 (1975).

[2] For a review see: A. Accardi, hep-ph/0212148 and references therein.

[3] See for example: X-N. Wang, Phys. Rev. C61, 064910 (2000).

[4] G. Papp, P. Levai and G. Fai, Phys. Rev. C61, 021902 (2000); A. Accardi and M. Gyulassy, Phys. Lett. B586, 244 (2004); E. Cattaruzza, D. Treleani, Phys. Rev. D69, 094006 (2004).

[5] B. Z. Kopeliovich, J. Nemchik, A. Schaefer and A.V. Tarasov, Phys. Rev. Lett. 88, 232303 (2002).

[6] R. C. Hwa and C. B. Yang, Phys. Rev. Lett. 93, 082302 (2004); R. C. Hwa and C. B. Yang, Phys. Rev. C70, 037901 (2004).

[7] For example: D. Kharzeev, Y. V. Kovchegov and K. Tuchin, Phys. Lett. B599, 23 (2004); J. Jalilian-Marian, Y. Nara and R. Venugopalan, Phys. Lett. B577, 54 (2003); J. P. Blaizot, F. Gelis and R. Venugopalan, Nucl. Phys. A743, 13 (2004); D. Kharzeev, Yu. K., K. Tuchin, Phys.Rev. D68, 094013 (2003); Albacete, Armesto, Kovner, Salgado and Wiedemann, Phys. Rev. Lett. 92, 082001 (2004); E. Iancu, K. Itakura and D. N. Triantafyllopoulos, Nucl. Phys. A742,182 (2004); J. Jalilian-Marian, Nucl. Phys. A748, 664 (2005).

[8] B. Z. Kopeliovich, J. Nemchik, I. K.Potashnikova, M. B. Johnson, I. Schmidt, Phys. Rev. C72, 054606 (2005); J. Nemchik, V. Petracek, I. K. Potashnikova and M. Sumbera, Phys. Rev. C78, 025213 (2008).

[9] N. Armesto et al., J. Phys. G35, 054001 (2008).

[10] J. Adams et al., (STAR Collaboration), Phys. Lett. B637, 161 (2006). 
[11] B. I. Abelev et al., (STAR Collaboration), Phys. Rev. Lett. 97, 152301 (2006).

[12] For example see: I. Vitev and M. Gyulassy, Phys. Rev. C65, 041902 (2002); R. J. Fries, B. Muller, C. Nonaka and S. A. Bass, Phys. Rev. C68 044902 (2003).

[13] D. C. Hom et al., Phys. Rev. Lett. 37, 1374 (1976); D. M. Kaplan et al., ibid., 40, 435 (1978).

[14] J. Huston et al., Phys. Rev. D51, 6139 (1995).

[15] L. Apanasevich et al., Phys. Rev. D59, 074007 (1999).

[16] M. N. Mangano, P. Nason, and G. Ridolfi, Nucl. Phys. B373, 295 (1992).

[17] J. C. Collins, Nucl. Phys. B396, 161 (1993); Phys. Lett. B536, 43 (2002).

[18] R. P. Feynman, R. D. Field and G. C. Fox, Phys. Rev. D18, 3320 (1978); For a review see: J. F. Owens, Rev. Mod. Phys. 59, 465 (1987).

[19] A. D. Martin, R. G. Roberts, W. J. Stirling and R. S. Thorne, Eur. Phys. J. C4, 463 (1998); A. D. Martin, W. J. Stirling, R. S. Thorne, and G. Watt, Phys. Lett. B 652, 292 (2007).

[20] S. Kretzer, Phys. Rev. D 62, 054001 (2000).

[21] S. Albino, B. A. Kniehl and G. Kramer, Nucl. Phys. B803, 42 (2008).

[22] B. Z. Kopeliovich, A. H. Rezaeian, H. J. Pirner and I. Schmidt, Phys. Lett. B653, 210 (2007); A. H. Rezaeian et al., arXiv:0707.2040.

[23] S. S. Adler et al., (PHENIX Collaboration), Phys. Rev. Lett. 98, 172302 (2007).

[24] B. Z. Kopeliovich, A. H. Rezaeian and I. Schmidt, Phys. Rev. D78, 114009 (2008) arXiv:0809.4327.

[25] K. J. Eskola, V. J. Kolhinen and P. V. Ruuskanen, Nucl. Phys. B535, 351 (1998); K. J. Eskola, V. J. Kolhinen and C. A. Salgado, Eur. Phys. J. C9, 61 (1999).

[26] M. Hirai, S. Kumano and T. H. Nagai, Phys. Rev. C76, 065207 (2007)

[27] S-y. Li and X.-N. Wang, Phys. Lett. B527, 85 (2002).

[28] M. B. Johnson, B. Z. Kopeliovich and A. V. Tarasov, Phys. Rev. C63, 035203 (2001).

[29] K. Golec-Biernat and M. Wüsthoff, Phys. Rev. D59, 014017 (1999).

[30] R. J. Glauber and G. Matthiae, Nucl. Phys. B21, 135 (1970); K. J. Eskola, K. Kajantie, J. Lindfors Nucl. Phys. B323, 37 (1989).

[31] M. B. Johnson et el., Phys. Rev. C65, 025203 (2002).

[32] N. Armesto, J. Phys. G32, R367 (2006) hep-ph/0604108].

[33] R. J. Fries, V. Greco and P. Sorensen, Ann. Rev. Nucl. Part. Sci. 58, 177 (2008) and references therein. 